\title{
Cloacogenic Polyps: An Unrecognized Cause of Rectal Bleeding
}

\author{
Joana Rita Carvalho ${ }^{1 *}$, Sofia Carvalhana ${ }^{1}$, Rita Luis ${ }^{2}$, Narcisa Fatela ${ }^{1}$, Rui Tato Marinho ${ }^{1}$, \\ Fernando Ramalho ${ }^{1}$, José Velosa ${ }^{1}$ \\ ${ }^{1}$ Department of Gastroenterology and Hepatology, North Lisbon Hospital Center, Portugal \\ ${ }^{2}$ Department of Pathology, North Lisbon Hospital Center, Portugal
}

Received: February 28, 2016; Accepted: April 14, 2016; Published: April 22, 2016

*Corresponding author: Joana Rita Carvalho, MD, Department of Gastroenterology and Hepatology, North Lisbon Hospital Center, Portugal, Tel : 00351916489846, E-mail address: joana.rita.carvalho@gmail.com

\begin{abstract}
Cloacogenic inflammatory polyps correspond to a rare subtype of prolapsing mucosal polyps that arise around the anal transitional zone. It usually presents with rectal bleeding, tenesmus and altered bowel habits, and can mimic neoplasia. It is an unrecognized cause of hematochezia which may be overlooked at colonoscopy unless an endoscopic retroflexion maneuver is performed. Endoscopists should be aware of this entity. We present a case of rectal bleeding caused by an inflammatory cloacogenic polyp in a cirrhotic patient. To our knowledge, this represents the first report of this entity in a cirrhotic patient.
\end{abstract}

Keywords: Inflammatory Cloacogenic Polyp; Cirrhotic; Rectal Bleeding

\section{Introduction}

Inflammatory cloacogenic polyps (ICP) correspond to a polypoid mucosal prolapse occurring in an anorectal location [1]. Some authors, consider that this entity is included within the spectrumof 'polypoid mucosal prolapsed syndrome' [2]. Endoscopically, the polyps appeared to be well-circumscribed, hyperemic masses that contrasted sharply with normalappearing adjacent mucosa [3]. ICP may be overlooked at colonoscopy unless an endoscopic retroflexion maneuver is performed [4]. Histological features include glandular crypt abnormalities, fibromuscular obliteration of the lamina propria, and thickened and splayed muscularis mucosa [1,3]. ICP are rare and usually benign, although there have been associated with anal neoplasias as well as squamous cell carcinoma [5]. Generally presents with rectal bleeding and in some cases tenesmus, [2, 6] but $20 \%$ of the patients are asymptomatic [3]. We present a case of rectal bleeding caused by an inflammatory cloacogenic polyp in a cirrhotic patient.

\section{Case Report}

A 58-year-old male patient was admitted due to decompensated cirrhosis with ascitis in the setting of an infected chronic diabetic ulcer. He had a medical history of alcoholic cirrhosis (abstinent for 1 year), portal hipertension, internal hemorrhoids (with occasional bleeding) and type II diabetes mellitus with a chronic ulcer of the letf leg. He was medicated with furosemide $80 \mathrm{mg} /$ day, spironolactone $150 \mathrm{mg} /$ day, omeprazole $20 \mathrm{mg} /$ day, propranolol $20 \mathrm{mg} /$ day and metformin $1000 \mathrm{mg} /$ day. On physical examination he was normotensive, febrile $\left(39.2^{\circ} \mathrm{C}\right)$, without signs of encephalopathy. Cardiopulmonary auscultation had no relevant findings and abdominal examination revealed non painful moderate ascites. A chronic ulcer of the left leg with signs of infection was observed. The laboratory findings revealed anemia (hemoglobin $11.2 \mathrm{~g} / \mathrm{dl}$ ), leukocytosis 10200/L, C-reactive-protein $8.2 \mathrm{mg} / \mathrm{dl}$, total bilirubin $2.8 \mathrm{mg} / \mathrm{dL}$, creatinine $1.1 \mathrm{mg} / \mathrm{dL}$ and INR 2.1. He was a Child-Pugh score of 10 (class C) and a MELD score of 20. Diagnostic paracentesis excluded spontaneous bacterial peritonitis and the patient started amoxicillin and clavulanic acid to the cutaneous infection. At fifth day of admission, the patient had red blood hematochezia with hypotension and loss of $2 \mathrm{~g} / \mathrm{dL}$ of hemoglobin. He had no abdominal pain or proctalgia. Nasogastric aspirate was bilious without blood. Upper gastrointestinal endoscopy was performed and revealed large esophageal varices in the distal oesophagus without red spots or stigmata of recent bleeding (figure 1A); stomach presented signs of mild hypertensive gastropathy (figure 1B and 1C). No blood was seen in the stomach, esophagus or duodenum(figure 1D). A first attempt of colonoscopy was made but was inconclusive due to red blood in the rectal lumen. After $24 \mathrm{~h}$ of this first attempt, total colonoscopy was performed. There was no blood in all colonic segments. A $10 \mathrm{~cm}$ pedunculated polyp without erosion or spontaneous bleeding was visible in the anorectal transition (figure 2A) and internal congestive hemorrhoids were also seen (figure 2B). Polypectomy was not performed. Three days after this episode, the patient presented once again with red blood hematochezia. Second look upper gastrointestinal endoscopy revealed the same aspects, without any bleeding lesions. Videocapsule was also performed and did not reveal any bleeding lesions of the small bowel. Colonoscopy was repeated and, again, the only finding was the pedunculated polyp mentioned above without stigmata of recent bleeding. Polypectomy was performed at this time. After this procedure, there were no more visible blood losses and hemoglobin values remain stable. Histopathologic analysis revealed a colonic polyp with mild fibromuscular hyperplasia of the lamina propria, with scattered "diamond" shaped crypts and indirect signs of previous ulceration, which was compatible with an inflammatory cloacogenic polyp (figure 3). 


\section{Discussion}

ICP is a rare type of anorectal polyp first described in 1981 [5]. ICP arises from the epithelial transition zone at the anorectal junction and have a distinctive histological appearance [5]. ICP corresponds to a polypoid mucosal prolapse that occurs in the anorectal area and is distinguished by ulceration and erosion on the surface [1]. Other features may include villiform hyperplasia, crypt architectural disarray, fibromuscular proliferation within the lamina propria, and variable degrees of chronic inflammation $[1,5]$. There is no set age of occurrence for these lesions, and cases have appeared in adults as well as children [5], although it is more common between the fourth and the sixth decades [3]. The exact pathophysiological mechanism behind this subtype of polyps remain unknown [2]. Some authors suggest that the combination of venous congestion and spastic contraction of the mucosae cause repeated ischemia and regeneration phenomena that could result in the ICP formation [7]. As mucosal prolapse accompanies $50 \%$ of reported ICP cases, it has been proposed that ICP is due to mucosal prolapse $[1,7]$. Endoscopically, ICP may
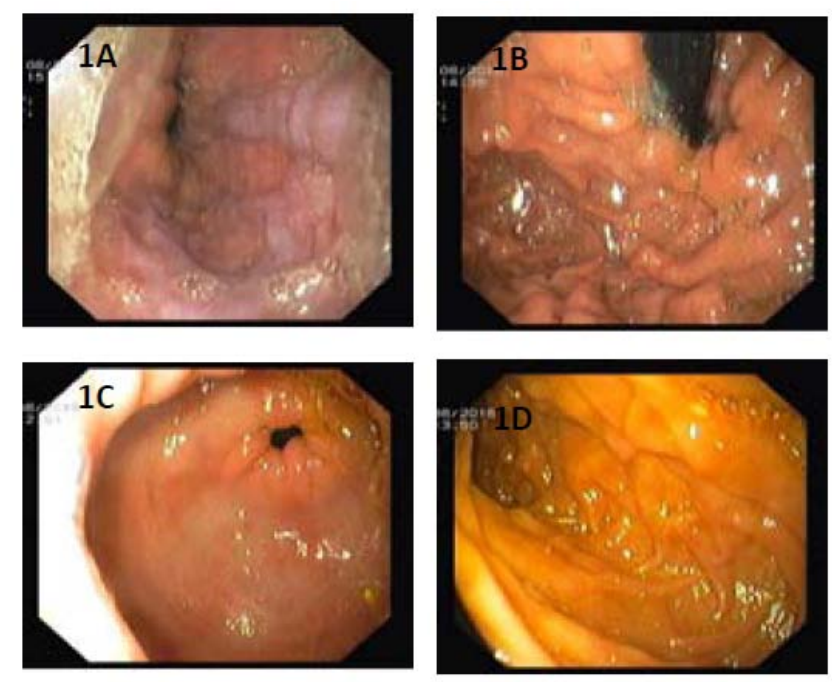

Figure 1: Upper gastrointestinal endoscopy showing large esophageal varices in the distal esophagus without red spots or stigmata of recent bleeding $(1 \mathrm{~A})$; stomach presenting signs of mild hypertensive gastropathy (1B and $1 \mathrm{C})$. No blood was seen in the stomach, esophagus or duodenum(1D).

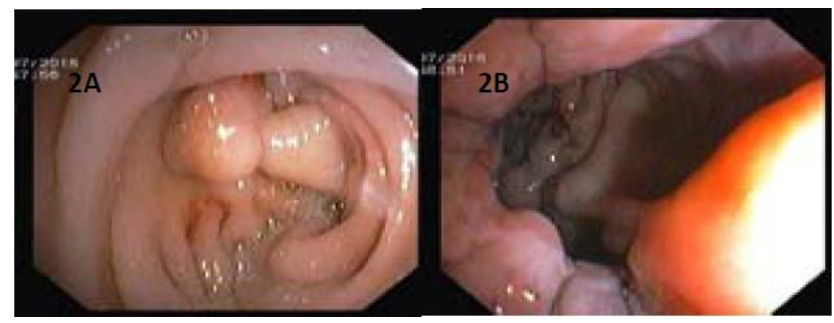

Figure 2: Colonoscopy showing a $10 \mathrm{~cm}$ pedunculated polyp without erosion or spontaneous bleeding in the anorectal transition $(2 \mathrm{~A})$ and internal congestive hemorrhoids (2B).

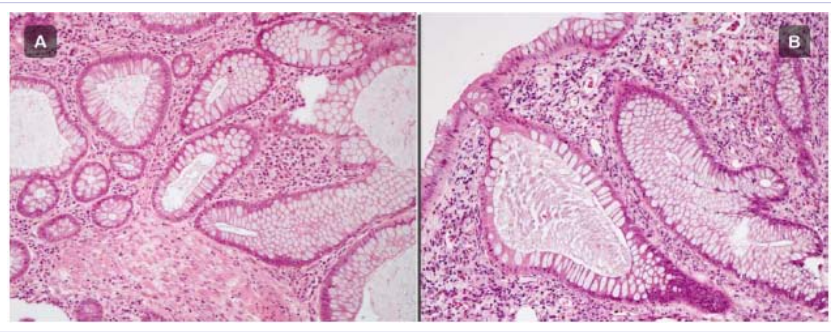

Figure 3: Hematoxylin and eosin stain, x100). (3A) Thickened smooth muscle fibers outstretch throughout the lamina propria, enveloping the colonic crypts and giving rise to the so-called "diamond" shapes. (3B) Hemosiderin deposits, granulation tissue and slight serration of the overlying epithelium herald previous ulceration.

appear as a villiform, tumorous mass at the anorectal junction [1] that may be pedunculated or more commonly sessile [5]. To the histopathologist the marked regenerative aspects may cause bewilderment with a villous adenoma or mimic anorectal cancer [1]. The most common symptoms of ICP are rectal bleeding, straining to defecate, swelling of the anus and pruritis[5]. ICP are classically associated with hemorrhoids and local prolapse $[1,5]$. In this case, the patient also presented with internal congested hemorrhoids. The rectal bleeding was also probably more exuberant in this patient, due to the coagulopathy associated to a decompensated cirrhosis. As far as we know, no association between ICP and cirrhosis has been reported. Treatment consists in endoscopic or surgical resection of the symptomatic ICP [3]. A high fiber diet has also been associated with regression of ICP in some patients [8].

To conclude, ICP represents one of the polypoid lesions that can be found in colonoscopy and endoscopists should be able to recognize it. It is also a very rare cause of hematochezia which may be underdiagnosed at colonoscopy unless an endoscopic retroflexion maneuver is performed. To our knowledge, this represents the first report of ICP as cause of bleeding in a cirrhotic patient.

\section{References}

1. Parfitt JR, Shepherd NA. Polypoid mucosal prolapsed complicating low rectal adenomas: beware the inflammatory cloacogenic polyp. Histopathology. 2008;53(1):91-96. doi: 10.1111/j.13652559.2008.03035.x.

2. Ciriza de los Ríos C, Tomas Moro E, García Durán F, Jesus Alvarez Sanchez, Fernando Bermejo San Jose, et al. Polipos inflamatorios cloacogenicos: una causa inusual de sangrado rectal. Gastroenterol Hepatol. 2007;30(8):461-464.

3. Tendler DA, Aboudola S, Zacks JF, O’Brien MJ, Kelly CP. Prolapsing mucosal polyps: an underrecognized form of colonic polyp a clinicopathological study of 15 cases. Am J Gastroenterol. 2002;97(2):370-376.

4. Poon KK, Mills S, Booth IW, Murphy MS. Inflammatory cloacogenic polyp: An unrecognized cause of hematochezia and tenesmus in childhood. J Pediatr.1997;130(2):327-329.

5. Kalogerinis PT, Morfesis FA, Georgakila S,Georgakilas AG. Correction of 
anal prolapse associated with resolution of cloacogenic polyp lesions. Implications to anorectal cancer. J Biochem Tech. 2009;1(2):62-64.

6. Zaman S, Mistry P, Hendrickse C, Bowley DM. Cloacogenic polyps in an adolescent: A rare cause of rectal bleeding. Journal of Pediatric Surgery. 2008;48:e5-e7.

7. Du Boulay CE, Fairbrother J, Isaacson PG. Mucosal prolapse syndrome, a unifying concept for solitary ulcer syndrome and related disorders. J ClinPathol.1983;36(11):1264-1268.

8. Oriuchi T, Kinouchi Y, Kimura M, Hiwatashi N, Hayakawa T, Watanabe $\mathrm{H}$, et al. Successful treatment of cap polyposis by avoidance of intraluminal trauma. Am J Gastroenterol.2000;95(8):2095-2098. 